Western University

Scholarship@Western

Psychology Publications

Psychology Department

2012

Semantic and Associative Relations in Adolescents and Young Adults: Examining a Tenuous Dichotomy

Ken McRae

Saman Khalkhali

Mary Hare

Follow this and additional works at: https://ir.lib.uwo.ca/psychologypub

Part of the Psychology Commons

Citation of this paper:

McRae, Ken; Khalkhali, Saman; and Hare, Mary, "Semantic and Associative Relations in Adolescents and Young Adults: Examining a Tenuous Dichotomy" (2012). Psychology Publications. 115.

https://ir.lib.uwo.ca/psychologypub/115 


\title{
Semantic and Associative Relations in Adolescents and Young Adults: Examining a Tenuous Dichotomy
}

\author{
Ken McRae ${ }^{1}$, Saman Khalkhali ${ }^{1}$, \& Mary Hare $^{2}$ \\ ${ }^{1}$ Department of Psychology, University of Western Ontario, London, Canada \\ ${ }^{2}$ Department of Psychology, Bowling Green State University, Bowling Green, OH
}

McRae, K., Khalkhali, S., \& Hare, M. (2012). Semantic and associative relations: Examining a tenuous dichotomy. In V. F. Reyna, S. B. Chapman, M. R. Dougherty, \& J. Confrey (Eds.), The Adolescent Brain: Learning, Reasoning, and Decision Making (pp. 39-66). Washington, DC: APA.

Address Correspondence to:

Ken McRae

Department of Psychology

University of Western Ontario

London, Ontario, Canada

N6A 5C2

Phone: (519) 661-2111 x84688

Email:kenm@uwo.ca

Address Correspondence to Ken McRae, Department of Psychology, University of Western Ontario, London, Ontario, Canada, N6A 5C2. Phone: (519)661-2111 x84688Ｅmail: kenm@uwo.ca

This work was supported by a National Institutes of Health grant HD053136 to Ken McRae and Mary Hare, and by Natural Sciences and Engineering Council Grant OGP0155704 to Ken McRae. 


\begin{abstract}
The constructs of semantic and associative relatedness have played a prominent role in research on semantic memory because researchers have historically drawn on the distinction between these two types of relations when formulating theories, creating experimental conditions, and explaining empirical results. We argue that the binary distinction between semantics and association is rooted in a fundamental problem in how the two are defined and contrasted. Whereas semantic relatedness has typically been limited to category coordinates, associative relatedness has most often been operationalized using the word association task. We show that meaningful semantic relations between words/concepts certainly extend beyond category coordinates, that word association is driven primarily by meaningful semantic relations between cue and response words, and that non-meaningful, purely associative relations between words generally are not retained in memory. To illustrate these points, we discuss research on semantic priming, picture naming, and the Deese-Roediger-McDermott false memory paradigm. Furthermore, we describe how research on the development of mnemonic skills in adolescents supports our view. That is, adolescents do not learn arbitrary associations between words, but develop elaborative strategies for linking words by drawing on their rich knowledge of events and situations. In other words, adolescents use existing memories of meaningful relations to ground their memories for novel word pairs, even in an associative learning paradigm.
\end{abstract}

The term "semantic memory" is used to refer to people's memory for concepts and word meanings. An important aspect of understanding semantic memory concerns delineating the ways in which knowledge of word meaning is organized, and as such, a great deal of research has been aimed at providing insight into this issue. A key goal in this regard is to uncover the relations among concepts that are encoded in semantic memory. To this end, the constructs of semantic and associative relations have been central components of theories of the organization of semantic memory, and research comparing the two has provided a substantial amount of informative data that have furthered both theory development and empirical work. However, critical issues remain with regard to how semantic and associative relations have been defined and studied in semantic memory research, and how they might best be defined and studied in future research.

In an influential paper on the organization of human memory, Tulving (1972) noted an increased interest among some of his contemporaries in the kind of memory that underlies the seemingly effortless execution of skills such as language processing and memory access. Tulving's definition of semantic memory still nicely captures some commonly held views:

Semantic memory is the memory necessary for the use of language. It is a mental thesaurus, organized knowledge a person possesses about words and other verbal symbols, their meaning and referents, about relations among them, and about rules, formulas, and algorithms for the manipulation of these symbols, concepts, and relations. (p. 386)

Tulving also stated that "the relations among items in semantic memory are of much greater variety" (p. 388) than the relations among the contents of episodic memories, which he believed to be organized chiefly along spatio-temporal dimensions.

Since that time, a large number of theories and studies have focused on the contrast between semantic and associative relations because they are considered to be the two principle and distinguishable components of conceptual organization (Crutch \& Warrington, 2010; Fischler, 1977; Hutchison, 2002; Shelton \& Martin, 1992; Thompson-Schill, Kurtz, \& Gabrielli, 1998; Yee, Overton, \& Thompson-Schill, 2009). It has been a common working hypothesis in semantic memory research that these components are defined on orthogonal dimensions. Associative relatedness is defined typically in terms of stimulus-response combinations in a word association task (e.g., agony-pain; Nelson, McEvoy, \& Schreiber, 1998). In fact, Nelson et al.'s word association norms, although not the 
sole source of word association norms in the literature, have been the most often used operationalization of association in memory research for at least the past decade.

In contrast, semantic relatedness has typically been defined either as membership in the same superordinate category (e.g., horse-dog; Lupker, 1984), or as the degree to which the semantic features of two concepts overlap (horse-cow; Frenck-Mestre \& Beuno, 1999). Often these two measures are treated as essentially the same, and indeed both are based on closeness in a representational structure, although featural overlap is more of a continuous dimension than is shared category.

In this chapter, we outline our position concerning the relationship between association and meaning. Association in its general sense - spatial and temporal co-occurrence in the world and language - is an important driving force in learning, and this includes the formation of semantic representations. Furthermore, word association norms are an interesting and rich source of data. However, word associations on their own provide little if any insight into the relations that are encoded in semantic memory. Performance on word association norms is driven by meaningful semantic relations, and these relations are identifiable, and in many cases, quantifiable. We also argue that is not fruitful to attempt to understand semantic memory using a binary distinction between semantic similarity and word association (or even between semantic relatedness, broadly defined, vs. word association). On the one hand, the scope of semantic relations is much broader than similarity alone, and on the other hand, word associations are driven almost exclusively by semantic relations. Finally, a fruitful research strategy is to work toward understanding the relative importance or centrality of various types of semantic relations for various types of concepts. This approach, we believe, is the best path forward for understanding concepts and semantic memory.

To provide evidence for these ideas, and to couch our arguments, we focus on four areas of research in which the semantics-word association dichotomy has played a major role. Section 4.1 deals with experiments regarding picture-word facilitation and interference. Section 4.2 concerns the DeeseRoediger-McDermott false memory paradigm. Section 4.3 focuses on semantic priming. Finally, Section 4.4 describes research concerning how the ability to learn word pairs develops across adolescence, and how this development crucially hinges on semantic knowledge, and the ability to employ that knowledge to make associations meaningful.

\subsection{Goals}

Our goals in this chapter are as follows. First, we define the scope of what we mean by "semantically related". We contend that lexical concepts are meaningfully related to each other in diverse ways, and that an important aim of semantic memory research is to provide an empirical basis for the theoretical delineation of these semantic relations. Our second goal is to define "associatively related". In general, an association between two concepts is due to their referents' spatial and/or temporal co-occurrence in the real world and/or in language. In cognitive psychology experiments, however, association typically is operationalized in terms of the word association task. Over time, this empirical operationalization has become conflated with the theoretical notion of association. In this chapter, we generally distinguish between these two meanings by using "word association" when referring to the results from a word association task.

We argue, as others have before us, (Anisfeld \& Knapp, 1968; Brainerd, Yang, Reyna, Howe, \& Mills, 2008; Grossman \& Eagle, 1970) that word associations are best understood in terms of semantic relations. We contend that it is a fruitful research strategy to work toward understanding what drives responses in a word association task. This requires detailed analyses of the types of semantic relations that underlie word association performance. On our view, association, in terms of spatial and temporal co-occurrence, is a critical component of the process of acquiring lexical representations, but retained associations between concepts are almost always semantic in nature, and thus are encoded as semantic relations. A key task in investigating how lexical concepts are related to one another is to test what 
sorts of relations influence behavior of various types (see e.g., Estes, Golanka, \& Jones, in press; Kalenine, Peyrin, Pichat, Segebarth, Bonthoux, \& Baciu, 2009).

Although we distinguish between association and word association norms, we do not argue that word association norms are useless or irrelevant. We do argue, however, that because normative word associations are a product of semantic relations, one should explore the relations between a word and its strongest conceptual associates. Indeed, word association norms provide a valuable metric for studying the relations between lexical concepts, but we do not believe they should be viewed as arising from undifferentiated associations of varying strengths. Thus, the goal should not be to construct theories that rely on undifferentiated word association mechanisms.

The remainder of this chapter discusses implications of our view for existing and future research. A major point is that the practice of partitioning stimuli into associatively and semantically related sets of items is neither justified nor empirically fruitful in the long run. This argument has a number of implications. One is that many studies that have partitioned their stimuli in this way may need to be reconceptualized in terms of the types of information that were actually manipulated. This may not possible in many cases because studies using word-associated items typically intermingle hetergeneous semantic relations within their stimuli. Instead, then, we suggest a strategy for future research that emphasizes careful attention to the types of relation(s) being manipulated.

\section{Semantic Relatedness}

In a substantial amount of past research, semantic relatedness has been defined exclusively in terms of category co-ordinates, or featurally similar concepts, depending on the theoretical point of view (for reviews, see Hutchison, 2003; Lucas, 2000). Although shared category (or featural similarity) is important for many cognitive tasks, focusing exclusively on this relation has led to a rather narrow point of view when studying semantic relatedness. Indeed, it could be argued that it has hindered progress on understanding semantic relations and semantic memory.

In contrast, some researchers have studied a number of ways in which lexical concepts are related to one another. That is, there are a large number of types of semantic relations that are rooted in something other than membership in the same category. We present a taxonomy of the various dimensions of semantic relatedness in Table 1, to give an indication of the types of relations that might be studied. Given these numerous relation types, the task of researchers is to theoretically and empirically delineate among them. In the course of doing so, it may be found that some of them are accessed rapidly and automatically, even from single words, whereas others either are not encoded in semantic memory, or are less strongly instantiated, thus requiring additional context (such as sentence, discourse, or real-world context) for their influence to be observed. Studies involving a number of these relation types are described briefly in Section 4.

\section{Associative Relatedness}

Association has a long history in psychology, and an even longer one in philosophy. Lexical association is a construct that is often used to explain performance in memory and psycholinguistic studies. Bower (2000) defines associations as "sensations that are experienced contiguously in time and/or space. The memory that sensory quality or event A was experienced together with, or immediately preceding, sensory quality or event B is recorded in the memory bank as an association from idea $a$ to idea $b . "$ (p. 3). Deese (1965), a crucial bridge between the methods of behaviorism and cognitivism, noted that "almost all the basic propositions of current association theory derive from the sequential nature of events in human experience" (p. 1). Moss, Ostrin, Tyler, and Marslen-Wilson (1995) suggested that associations between words themselves are "built up through repeated cooccurrence of the two word forms." (p. 864). Fischler (1977) argued that associations between words could be formed from "accidents of contiguity," leading to the methodology, still used today, of 
separating stimuli into associated versus purely semantically related groups. Thus, the consensus regarding association appears to be that contiguity is key to forming a link between two concepts.

However, despite the consensus concerning the importance of contiguity, association in cognitive psychology typically is defined in terms of its operationalization, word association norms. In a word association task, a stimulus word is presented to a participant, who then produces the first word that comes to mind. Note that this operationalization is far removed from both the classical and modern definitions of association. Two words are now associated if one is given as a response to the other in a word association task. There are a number of discontinuities between the definition of association and its operationalization. Association proper is learning-based; word association is retrieval or productionbased. Association proper is based heavily on sensory information; word association is linguistically based. Association is based on contiguity, accidental or otherwise; word associations are, as we show, almost always meaningful.

Many researchers have used word association, often taken from Nelson et al.'s (1998) norms, to predict or explain performance on semantic and memory tasks. Typically, the responses are interpreted as being part of an associative network that predicts fast, automatic processing. However, much less research has attempted to determine the source of particular associative relations between two concepts (although doing so dates back at least to Jung, 1919). For instance, if hammer and nail are strong associates (Nelson et al.), then why do participants respond nail to hammer? In an attempt to answer this question, Santos, Chaigneau, Simmons, and Barsalou (in press) classified word associations based on the relationship between the stimulus and response. Their classification taxonomy is presented in Table 2 .

This taxonomy reveals the semantic nature of most word associations. Even those categories based on compound words or phrases can, in some sense, be considered as a semantic relationship. The first category is compound word continuation (fruit-fly, and bus-boy), with this type of item having been used to test for associative priming (Thompson-Schill et al., 1998; Yee et al., 2009). However, the fact that each of the words in the pair can stand on its own as a separate word may be irrelevant; a fruit fly is a distinct semantic concept, different in representation from a horse fly, a housefly, a butterfly, and even just a fly, which as a noun usually refers to a housefly. Therefore, it is not an accident of contiguity that these two words are related; they are instead used in conjunction to refer to a specific concept. The second type of association in Santos et al.'s taxonomy is reversed compound words, such as fly-fruit. The third category is sound similarity. This is the least semantic of all the types of word associations. Root similarity is semantic (or at least morphological) because different linguistic forms of the same concepts comprise the two members of the word pair.

It is difficult to dispute the semantic character of the final six categories in Santos et al.'s (in press) taxonomy. Synonyms and antonyms are highly semantically similar concepts, with antonyms differing on a single dimension of meaning, such as size (small-large). The next three categories deal with category membership: superordinates, subordinates, and category coordinates. These three types of word association are the most frequently used as semantically related items in cognitive experiments. Superordinate (chair-furniture), subordinate (insect-grasshopper) and category coordinate relations (robin-sparrow) were incorporated as semantic relations in early models such as the hierarchical model (Collins \& Quillian, 1969; although see O'Connor, Cree, \& McRae, 2009). The last category, aspect of an object or situation, is overly broad, so it may be more useful to separate object features and scenes. Featural relations for objects (bird-wing) are a crucial aspect of semantics. Situational relations (restaurant-menu) rely on the participant generating a scene, script, schema, or otherwise using the common contexts of the two concepts in the pair.

From the examples above, it is clear that at least six, and possibly as many as nine, of the ten proposed categories in Santos et al.'s (in press) taxonomy of word associations are semantic. They are not based on accidental contiguity in space or time. They are likewise not solely based on perceptionoriented associations. Instead, these word association categories can more easily be expressed in terms 
of semantic relations. The few that are not semantic may be better defined as linguistic relationships, with sound similarity representing phonological knowledge, and root similarity arising from morphological information. Finally, another excellent example of classifying the semantic aspects of word association norms can be found in Guida and Lenci (2007), who focused on norms for Italian verbs.

Association has long been recognized as being crucial to the establishment of semantic representations (Nelson, McEvoy, \& Dennis, 2000), but no consensus has been reached regarding the process by which this transformation from association to semantic relation occurs. Computational models have been introduced, based on large corpora of text and/or speech (Burgess \& Lund, 1997; Jones, Kintsch, \& Mewhort, 2006; Landauer \& Dumais, 1997; Riordan \& Jones, 2011), but these theories have frequently met considerable opposition because of the purely linguistic nature of the associations involved in their computations, which is ironic considering the source of word association data. Nevertheless, issues remain concerning how the individual episodes that are associations become assimilated into semantic memory. In his 1959 article, Deese worried that the structural aspects of associative information were being lost in his contemporaries' concentration on temporal and spatial contiguity. In his 1965 book, Deese argued that associative meaning does not imply association in the behaviorist sense (i.e., focused on temporal contiguity). Rather, it is a natural distribution of responses to a particular stimulus. He described two main characteristics of associative meaning, 1) the limitation of these associations to verbal responses, and 2) the minimization of contextual influence on these responses. Deese argued that the subsequent distribution of responses to a word stimulus thus defines the meaning of that word. This suggests that associative meaning is a subset of meaning proper, but it is the only analysis of meaning possible given the limitations of word association. Thus, whereas associations in similar experiments performed today are typically limited to the most frequent few responses, Deese's framework for associative meaning incorporated all potential responses to a given stimulus.

Deese (1965) did not specify a transformation from association to semantic knowledge, but he did argue that the complete set of associations to a word comprises the semantics of that word. This is not dissimilar to the view of semantics proposed in this chapter. We argue that a sufficiently large set of associations, plus an understanding of the underlying semantic relations, minus relations such as phonological similarity, would likely result in a reasonable first-order approximation of a lexical concept. We have taken advantage of this proxy in past research. That is, semantic feature production norms, as collected by McRae, Cree, Seidenberg, and McNorgan (2005) or Vinson and Vigliocco (2008), can be viewed as a form of word association (this is another example of the fuzziness of the distinction between a semantic task and a typical word association task). However, in feature production norms, participants are directed to produce semantic relations of various sorts, and responses are classified into types of semantic relations.

One counter-argument to the assertion that all retained associations are meaningful concerns experiments in which associations are learned between non-related concepts or nonsense words (Berry $\&$ Cole, 1973). Evidence of such learning would support the notion that even meaningless associations are retained, and therefore associations need not be semantic. However, it is unclear whether meaningless associations actually are retained. It appears that meaningless associations are in fact quite brittle and decay rapidly after the initial learning and testing phase unless an effort is made to make them systematic and meaningful, such as by incorporating them into a sentential context (Prior \& Bentin, 2003, 2008). Furthermore, meaningless associations generalize in a limited fashion, only influencing processing if the testing phase and the learning phase present the associated stimuli in an identical or close-to-identical fashion (Goshen-Gottstein \& Moscovitch, 1995; Pecher \& Raaijmakers, 1999). Finally, even when detected, the effect seems to require extensive training over the course of several days (Schrijnemakers \& Raaijmakers, 1997) or weeks (Dagenbach, Horst, \& Carr, 1990), and several studies have failed to detect any effect (Carroll \& Kirsner, 1982; Neely \& Durgunoglu, 1985; 
Smith, MacLeod, Bain \& Hoppe, 1989). It appears then that meaningless associations are not retained precisely because they are meaningless. That is, these laboratory-learned associations are discarded because they are unimportant.

In summary, as a dependent variable, the distribution of responses in a word association task is interesting, informative, and has been used to account for findings in numerous experiments. However, the pattern of responses in a word association task does not inform researchers why some concepts are more or less associated with others. Certainly, investigations of word association data show that the semantic relations between cue words and their respective associates are not randomly determined. Certain types of semantic relations appear to play a key role in determining people's responses. That is, there may be many reasons for why two lexical concepts are associated, but word association by itself does not provide insight into this issue. As with semantic relations, a more nuanced notion of association emerges after examining the various ways in which words can co-occur in space and time. As Deese (1965, p. vii) stated, "The central thesis of this book asserts that what is important about associations is not what follows what, but how sets of associations define structured patterns of relations among ideas."

\section{Research Relying on Differentiating Between Semantic and Associative Relations}

\subsection{Picture-Word Facilitation and Interference}

The role of context in lexical selection and production fluency has often been studied by testing the effect of a visually presented word on the time it takes to name a previously, simultaneously, or subsequently displayed picture. For example, a picture of a cat might be presented for naming along with a word such as dog or meow. By altering the delay between presentation of the word and picture, researchers have investigated the time course of the influence of lexico-semantic processing of the word on semantic processing and subsequent naming of the picture.

Somewhat perplexingly, although some early experiments showed that the processing of semantically related words facilitates naming latencies for the target picture (Bajo, 1988; Sperber, McCauley, Ragain, \& Weil, 1979), others showed interference (Glaser \& Dungelhoff, 1984; Rosinski, 1977). To try to understand these inconsistent results, researchers have investigated the type of relation between the word and the picture. For example, La Heij, Dirkx, and Kramer (1990) suggested that categorical relations on their own do not cause facilitation. Rather, they argued that an additional associative relation exists between some category coordinates (cat-dog), and this is what yields priming. Their own experiments, which contrasted the effect of word primes that are both categorically and associatively related to the picture with the effect of categorically related but unassociated primes, supported this distinction between relations.

This point of view has been adopted by many other researchers. In the picture naming literature, a common finding is that categorically related context words interfere with picture naming. For example, reading $d o g$ increases the time it takes to name a picture of a mouse. Conversely, words that are associatively but not categorically related to the target picture have generally been found to facilitate picture naming, as in the case of reading cheese and producing "mouse." Thus, borne out of an effort to tease apart the sometimes subtle, sometimes obvious variations in the stimuli used to test picture-word facilitation and interference, the literature investigating this task appears to support a double dissociation between semantic and word associative relations. After describing two representative studies that are based on this distinction, we present arguments regarding the flaws inherent to such an approach.

As an illustrative example of how the semantics/association distinction is typically supported empirically, consider a study by Alario, Segui, and Ferrand (2000) who sought to clarify the role of semantic and associative processing in lexical production. The authors contrasted priming effects from semantically related word-picture pairs, such as thread-“rope", with effects from associatively related 
stimuli such as carrot-"rabbit." The authors used a number of stimulus onset asynchronies (SOA, the time between the presentation of the word and the picture) to test whether the influence of each relation on picture naming depends on the point in time at which the target picture is processed relative to the prime word. Crucially, Alario et al. claimed that they distinguished between the two relation types by ensuring that none of the category coordinate pairs were normatively associated, and by choosing associatively related stimuli that were not category coordinates. When the onset of the word preceded the picture by $234 \mathrm{~ms}$, no effect was obtained with category coordinates, whereas word associates facilitated picture naming. On the other hand, at a $114 \mathrm{~ms}$ SOA, category coordinates interfered with picture naming, but word associates had no effect. Note that the word and picture did not overlap in time in either case.

Alario et al. (2000) focused on addressing a particular debate in the literature on lexical selection, namely whether there is competition between the lexical representations corresponding to the concept shown in the picture and similar concepts. That is, how does the (node corresponding to the) name of an object concept become uniquely selected for vocalization when naming a picture? Alario et al. concluded that interference effects are due to competition between semantically similar candidates, whereas associatively related words do not interfere with naming at any SOA because they are not lexical competitors of the picture name. That is, carrot does not compete with rabbit during production. The facilitation produced by word associates, on the other hand, was attributed to spreading activation from the word prime's lexical node to the node corresponding to the target picture in a localist production lexicon. In the Alario et al.'s view, the separate loci of facilitation and interference effects constitute a dissociation between the mental processes that support each of the two types of relations.

Similar reasoning has been used to argue in favor of a semantic-associative distinction, using a variation of the conventional picture naming paradigm. In the picture-word interference paradigm, a word is displayed at a location on the screen overlapping with a picture at some time before, after, or at the same time as, the onset of the picture. The key difference between the picture-word interference task and the paradigm used in Alario et al. (2000) is that in the former case the distractor word stays on screen following its presentation, and so is superimposed on the picture. In a recent study employing this paradigm, category coordinate distractors such as raisin - "apple" interfered with picture naming when they were displayed 150 or $0 \mathrm{~ms}$ prior to onset of the picture (Sailor, Brooks, Bruening, SeigerGardner, and Guterman, 2009). In contrast, word associates such as pie - "apple" facilitated picture naming when presented between 450 and $0 \mathrm{~ms}$ prior to picture onset. While they did not draw strong conclusions regarding a definite dissociation between associative and semantic relations, Sailor et al. suggested that "the facilitatory influence of associates occurs at a different stage of picture naming than the [interfering] influence of coordinates" (p. 797), in line with Alario et al.'s reasoning.

While we certainly do not doubt the importance of using the picture naming paradigm to probe the mental representations and processes underlying speech production and semantic memory, we suggest that the frequently proposed binary distinction between semantic and associative relatedness is not supported by empirical results. In particular, we argue that there is no basis for positing a double dissociation between associative and semantic priming effects. The fact that word associative and semantic relatedness are defined according to different operationalizations does not guarantee their independence.

The issue revolves around the architectural distinction between semantic and associative relatedness that seems overly narrow and ill-defined. As we discussed above, there is no clear consensus on where the boundary lies. Although category membership is the most frequently used operationalization of semantic relatedness, some researchers also recognize that other kinds of relations fall under this broad heading. Associative relatedness suffers from somewhat different problems. In some studies, associates were chosen using existing word association norms (Alario et al., 2000, Sailor et al., 2009), whereas in other studies, participants rated the degree of association between two words (Bolte, Jorschick, \& Zwisterlood, 2003). 
We argue that distilling relatedness into associative and semantic components is not a viable strategy for attaining a deeper understanding of the processes involved in picture naming. Recent work shows the relationship to be more graded than that. With regard to semantic relations, priming between semantically similar concepts can be explained in terms of feature overlap. Indeed, Vigliocco, Vinson, Lewis, and Garrett (2004) tested the influence of degree of semantic similarity based on their Featural and Unitary Semantic System (FUSS) model, which incorporates feature-based representations of concepts. The research nicely illustrates the graded effects of semantic similarity on picture-word interference for both object nouns and action verbs. This type of research, in which a specific semantic relation was identified and tested, is precisely the type that we advocate. Nonetheless, there are many other types of semantic relations yet to be explored.

As for associative relations, priming between other kinds of meaningfully related words has not been adequately explained except by appealing to spreading activation. What appears to be needed is a taxonomy of the various ways in which primes/distractors can be meaningfully related to targets, to test and understand the microstructure of semantic interference/facilitation effects, and their implications for the structure of semantic memory and for word production.

In fact, Costa, Alario, and Caramazza (2005) have made arguments along these lines. As they state, "it is unclear whether one can compare coordinate relationships to associative relationships. This is because associative relationships are heterogeneous: Whereas some associates are clearly semantically related, others are not" (p. 126). Using a picture-word interference task, they compared two types of semantic relations, "has a" relations (i.e., part-whole relations: stinger - "wasp"), and category coordinates (bee - "wasp"). Costa et al. found that parts facilitate picture naming, whereas category coordinates inhibit it. They based their interpretation on spreading activation networks. When the distractor is a semantically related part or category coordinate, spreading activation from the distractor to the semantically related target increases the activation of the target's lexical node (which by itself should cause facilitation). However, because the category coordinate belongs to the same superordinate category as the target, it is a possible candidate for selection in production, which causes interference in choosing the correct name. In contrast, activation of a "has a" distractor node is quickly disqualified as a possibility for selection, because it belongs to a different type of relation. No interference occurs, and facilitation is observed because of initial activation of the target. Although we would not use this type of spreading activation metaphor to account for results such as these, we do believe that this type of research moves toward understanding the influence of specific types of semantic relations.

One could imagine probing more deeply into types of relations, and other aspects of concepts. For example, would typical parts (wheels-truck), functions (transportation-truck), or locations (garagetruck) produce equivalent or different results? Perhaps aspects of concepts might show differential effects if the prime word denotes information from various modalities. Would distinctive features of concepts (moos - "cow") produce equivalent facilitation to that produced by features that are shared among multiple concepts (chews - "cow")? Studies such as these may be ultimately more informative about the structure and complexity of semantic memory than a simple (and somewhat artificial) bifurcation into semantic versus associative relations.

\subsection{Deese-Roediger-McDermott False Memory Paradigm}

The Deese-Roediger-McDermott (DRM) false memory paradigm has been used in numerous experiments to investigate representations and processes underlying false recall and recognition. In DRM experiments, participants typically are presented with a list of about 15 words, and then are asked to recall them, and possibly given a recognition task as well. The proportion of participants who falsely recall or recognize a critical non-presented word is measured. For example, the list for the critical nonpresented word doctor consists of nurse, sick, lawyer, medicine, health, hospital, dentist, physician, ill, patient, office, stethoscope, surgeon, clinic, and cure. 
The distinction between semantic and word associative relations has played a major role in this research for two reasons. First, word association explanations of DRM false memories enjoy a somewhat privileged position because, dating back to Deese (1959), DRM lists have been constructed using word association norms in the vast majority of studies. For example, many lists that have been used in DRM research, such as the doctor list presented above, consist of the 15 strongest word associates to the critical word. Second, the two major theories of false memories differ with respect to the centrality of associative and semantic relations. Activation/monitoring theory is based on spreading activation in a semantic network, and therefore the strength of normative association between list words and the non-presented critical word has played a major role in accounting for DRM false memories in this framework (Roediger, Watson, McDermott, \& Gallo, 2001). For example, Roediger et al. present word association and false recall and recognition data for a set of 55 commonly used DRM lists. Using regression analyses, they showed that the degree of association from the list words back to the critical word (backward associative strength, or BAS) strongly predicts the probability of false recall and recognition across those 55 lists.

The contrasting major framework is fuzzy-trace theory (Brainerd \& Reyna, 2002; Payne, Elie, Blackwell, \& Neuschatz, 1996). The primary assumptions of this theory are that a verbatim trace and a gist trace are produced during encoding, and the corresponding processes operate in parallel. The verbatim trace represents the surface form of the presented list items, and the gist trace represents the semantic content, including the list words' meanings, and the semantic relations among items (Brainerd \& Reyna, 2002). False recall is due to gist extraction during encoding, whereas veridical recall is due to verbatim traces. That is, in fuzzy-trace theory, gist extraction (or episodic interpretation) of the semantic content of, and the semantic relations among, the list words serve as the primary source of false memories. Therefore, this theory leads researchers to investigate the semantic content of the associatively-derived DRM lists.

One challenge for such semantic relations-based theories of false memories is to demonstrate that DRM effects that appear at first glance to be due to word associations are actually due to semantic relations. Brainerd et al. (2008) and Cann, McRae, and Katz (in press) have provided such evidence. Cann et al. used a knowledge type taxonomy developed by Wu and Barsalou (2009) to classify the relations found in the 55 commonly used DRM lists (Roediger et al., 2001). They classified list items into aspects of a situation in which the critical non-presented concept takes part (music-concert, with the first item being the critical concept, and the second a concept from the list), synonyms (trashgarbage), antonyms (beautiful-ugly), taxonomic relations (fruit-apple), entity relations (window-glass), and introspective relations (needle-hurt). Cann et al. found that virtually all of the words on DRM lists could be classified into these relations, and that the number of items of certain relation types predicted mean backward association of the DRM lists. In particular, BAS was related to the number of situation relations, synonyms, antonyms, and taxonomic relations on a list. In addition, Cann et al. found that the number of situation relations, synonyms, entity relations, and taxonomic relations predict the probability of false recall. Finally, they demonstrated that lists of words that consist only of situation relations produce high rates of false recognition even though BAS is essentially zero. For example, their breakfast list had a mean BAS of only .03, and contained bacon, cereal, food, coffee, eggs, fruit, juice, milk, pancakes, plate, muffin, and toast.

Brainerd et al. (2008) presented an overlapping but more detailed investigation into the semantic content of DRM lists and of BAS in general. They investigated 16 semantic properties, and found that DRM lists were exceptionally rich in meaning. These included the seven dimensions of Toglia and Battig's (1978) semantic word norms (familiarity, meaningfulness, concreteness, imagery, categorizability, number of attributes, pleasantness), the three dimensions of Bradley and Lang's (1999) emotion word norms (arousal, dominance, valence), the $\mathrm{Wu}$ and Barsalou (2009) knowledge types from Cann et al. (in press), and the non-semantic predictors used by Roediger et al. (2001). 
Brainerd et al. (2008) conducted factor analyses to investigate the factors on which these variables load. For the 55 DRM lists from Roediger et al. (2001), they found them to be rich in terms of the semantic variables, both those that measure aspects of single words (such as meaningfulness) and those that measure semantic relations. Furthermore, in a factor analysis using Roediger et al.'s data, false recall, false recognition, non-presented critical word familiarity, meaningfulness, and number of attributes, as well as mean BAS all loaded on one factor. In an analysis using false recognition data from their study in which participants did not first recall the list, false recognition loaded on a factor with virtually the same semantic variables.

Brainerd et al. (2008) also constructed a stratified sample of 400 cue-target word pairs, using Nelson et al.'s (1998) norms to obviate any concerns regarding the manner in which DRM lists are constructed and constrained. Importantly, a number of the semantic variables varied by cue-target association strength. Considering the semantic properties of the target words, increases in cue-target association strength were accompanied by higher levels of categorizability, concreteness, familiarity, imagery, meaningfulness, number of attributes, and pleasantness, and decreases in arousal and dominance. Considering the properties measured for cue words, increases in association strength were accompanied by increases in concreteness, imagery, and categorizability, but decreases in valence and dominance. Finally, in terms of cue-target semantic relations, increases in association strength were accompanied by increases in synonyms, antonyms, and taxonomic relations, as well as decreases in introspective and situational relations.

In summary, research into the DRM false memory paradigm is an example of an area in which undifferentiated word associative relations has played a central role. However, recent research demonstrates that false memories can arise in the virtual absence of word association. Furthermore, the key associative variable, BAS, can be understood in terms of semantic variables and relations.

\subsection{Semantic Priming}

An asymmetry exists between the treatment of word association and semantic relations in the semantic priming literature. This is perhaps due to the assumed bottom-up nature of associations and the top-down nature of semantics, or perhaps to the fact that association has a much longer history. In semantic priming research, it is typical to remove associated pairs from semantically related stimuli, but rare to strip semantic relatedness from associated pairs. However, this masks an even deeper asymmetry in the treatment of the two constructs; it has simply been assumed that associative priming exists. From the first attempts to remove association from semantic stimuli (Fischler, 1977), and the first models of semantic relatedness (Collins \& Loftus, 1975, Collins \& Quillian, 1969), there has been an implicit assumption that activation spreads through associative links, and therefore if one is to study true semantic connections between concepts, it is necessary to first negate or partial out word association. Thus, researchers have investigated "pure semantic priming without association" to discern semantic organization. Indeed, several authors (Hutchison, 2003; Lupker, 1984; Shelton \& Martin, 1992) have argued that all so-called semantic priming is in fact associative. We believe that these researchers have come upon the same problem we discuss in this chapter, but from the opposite direction: the basic inability to distinguish what is considered associative from what is considered semantic.

The typical methodology for demonstrating priming based on semantic relations has been to omit all word pairs that are also associated according to word association norms (Fischler 1977). On the assumption that associatively related items have been removed, a "pure" semantic category is left, with word pairs that are usually members of the same category (such as bear - cow). Although it has occasionally been found that this process eliminates priming (Lupker, 1984; Shelton \& Martin, 1992), the consensus now appears to be that semantic priming remains intact (Chiarello et al., 1990; Hare, Jones, Thomson, Kelly, \& McRae, 2009; Hines, Czerwinski, Sawyer, \& Dwyer, 1986; Seidenberg, Waters, Sanders \& Langer, 1984). "Pure" semantic priming has been found based on differesnt types of 
relations (similar concepts, verb-patient relations, event-based relations) even when this type of item filtering is performed. Therefore, pure semantic priming does exist.

Interestingly, there has been no such conclusive demonstration in the opposite direction. Very few researchers have attempted to find a "pure" associative priming effect, one in which all traces of semantic relationships have been removed from the stimuli. The studies that have attempted to do so have demonstrated how difficult - or perhaps impossible - this is. Many authors have tried to limit the effect of semantics on associative word pairs, but because the definition of semantic relatedness in these studies has usually been limited to category membership, many other semantic relationships have intruded into associatively related stimuli. A few examples are listed in Table 3. Some earlier experiments (Fischler, 1977; Lupker 1984) restricted their definition of semantics to category membership, which we argue is overly constrained. In those studies that did not, such as ThompsonSchill et al. (1998) and Yee et al. (2009), to remove semantic relations, they used compoundcontinuation forward items such as bell-boy and book-worm as their associatively related stimuli. However, they did not find priming for such items.

Experiment 3 of McKoon and Ratcliff (1992) is likely the most successful attempt to date at showing an associative priming effect in the absence of semantic relations. Rather than relying on word association norms (though these were included as a baseline), they used corpora co-occurrence statistics to measure contiguity. The assumption was that if the words co-occurred more than at chance in a six-million word text corpus, then they should be "tagged" by the memory system as being associated through repeated contiguity. McKoon and Ratcliff found facilitation for high co-occurrence items, although the same was not true for lower (but still higher than chance) co-occurrence items. However, an examination of their stimuli reveals that many of the high co-occurrence items are, in fact, semantically related through scene relations (kitchen-knife; hospital-baby), category membership (air water), or featural information (young-kids; black-smoke). Others were compound continuation forward relationships, as in Thompson-Schill et al. (1998), such as movie-stars, apple-pie, heat-wave, firetrucks, and power-plant. There were very few semantically related items among their low-cooccurrence items, (although some existed, such as amputation-leg), but this condition did not produce priming.

These data again illustrate one of the main points of this chapter, that concept pairs that produce behavioral consequences are related in meaningful ways. In McKoon and Ratcliff (1992), there are reasons why words co-occurred in a systematic manner, and the majority of their high co-occurrence items are semantically related in obvious ways. Again, we argue that this is not a coincidence. Higherthan-chance levels of co-occurrence between words are meaningful, and the semantic system takes advantage of this systematicity.

To date, no study has conclusively shown that pure associative priming exists, at least using preexisting knowledge tapped either by word association norms or local co-occurrence in corpora. Semantic priming in the absence of word association, in contrast, has been demonstrated many times (Ferretti, McRae, \& Hatherell, 2001; Hare et al., 2009; McRae \& Boisvert, 1998; see Lucas, 2000, for a review). Therefore, we conclude that it is much more likely that semantic relations are responsible for all of the results seen in thirty years of semantic priming experiments, and that associative priming either simply does not exist, or is so fragile that any associations that are not incorporated into the semantic system are quickly lost.

This conclusion leads to a number of recommendations. The first is our strong recommendation that items should not be removed from semantic priming tasks because the target was produced as a response to the prime in a word association task. We have argued in this chapter that because these associative responses are driven by semantic processes, the net effect of removing word associates from semantically related stimuli is to weaken semantic priming and make it more difficult to detect an effect if one is present. The primary consequence of omitting word associates is to remove the best items from a particular type of relationship. For example, in semantically similar priming stimuli used 
in McRae, de Sa, and Seidenberg (1997), the word associated items had higher similarity ratings (5.4 on a 9-point scale) than did the non-associated items (4.8). This is the case because semantic similarity is one factor that drives responses in a word association task.

Although researchers rarely talk about such experiences in print, we provide an illustrative anecdotal example regarding why it is illogical to remove associates from semantic stimuli. Ferretti et al. (2001) investigated priming between verbs and their typical agents (arresting-cop), patients (stirredspoon), instruments (cut-knife) and locations (skated-arena). Such verb-noun pairs definitely should co-occur in sentences. We were aware that potential reviewers would likely demand removal of any items that were normatively associated. Therefore, we conducted analyses removing all word associates from our stimuli. The reviewers' comments were telling of the confusion that surrounds these issues. One reviewer essentially stated, "Responses in a word association task tend to come from the same major syntactic category as the stimulus, so your items are probably still associated." That is, even though they were not associated, the priming results were not valid because the items were probably still associated. However, another reviewer's comment was essentially that, "It seems silly to remove associated items according to word association norms. Of course arrest and cop or eat and fork are associated in the broad sense of the word, because they occur together in the world and in language. That's the point, isn't it?" We believe that this was exactly the point, in addition to Ferretti et al.'s goal of specifying the type of relationship in each condition and separately testing each type of relationship. That is, meaningful associations in the world and language are just that, and these associations are retained in memory as semantic knowledge.

In support of this position, we draw attention to Nelson et al.'s (1998) description of their instructions to participants in the word association task: "Participants were asked to write the first word that came to mind that was meaningfully related or strongly associated to the presented word on the blank shown next to each item." (our emphasis in italics). Clearly, the manner in which word association norms have been used theoretically to draw a clear distinction between associative and semantic relations does not coincide with the actual instructions given to participants. That is, there exists no definitive line between word association and semantic relatedness.

\subsection{Adolescents Use Meaning to Learn Associations}

Throughout this chapter, we have argued for the intrinsically semantic nature of learned, retained associative relations. Arbitrary associations are rarely retained because this requires at least some degree of meaningful relatedness. Interestingly, the ability to learn arbitrarily related word pairs increases across adolescence, suggesting that the ability to make them less arbitrary - to create or elaborate meaningful relations between them - develops across that period. Experimental findings, summarized below, are consistent with that suggestion.

Rohwer, Rabinowitz, and Dronkers (1982) were among the first to test this developmental trend. The authors created a set of word pairs that norming studies had shown to have either an 'accessible' relationship (ranch-cowboy) or an inaccessible one (ranch-floor), and used them as stimuli in a cuedrecall task. The participants were fifth and eleventh grade students. These age groups bracket the adolescent years, as fifth graders are generally 10-year-old preadolescents, and eleventh graders are typically 17 years old.

In Rohwer et al. (1982), half of the participants in each age group were prompted to elaborate as they heard the word pairs, whereas the other half were not. Preadolescents' performance was strongly influenced by the prompt to elaborate, but much more so in the inaccessible than the accessible condition. The average number of correct responses to inaccessible items (ranch-floor) increased from 15 to 25 when the children had been prompted to elaborate during the study phase. In contrast, the average number of correct responses to accessible items (ranch-cowboy), which was relatively high to begin with, showed a more modest rise following the elaboration prompt. 
Older adolescents, on the other hand, showed only an effect of accessibility. They made more correct responses to accessible than to inaccessible pairs in both prompt conditions, but their number of correct responses, even to the inaccessible pairs, was significantly higher than that of the younger children.

To account for these age-related differences across adolescence, the authors argued that the children could learn the arbitrary pairs only if they made them less arbitrary - that is, if they elaborated a relationship between them. Rohwer et al. (1982) noted that in order to do this, the child must first develop sufficient knowledge of common events, because without it, she will have nothing on which to base a relationship. On this view, then, the developmental trend in paired-associate learning involves two factors. One is processing ability, or what the authors refer to as the propensity to elaborate. But as they point out, even with the highest ability, the child cannot elaborate an appropriate relationship unless she has developed a database of relevant events to use as a template. The younger children, then, were unable to learn the inaccessible pairs because they had insufficient knowledge of events to enable them to generate one that included both members of the pair.

Children also seem to develop more sophisticated learning strategies across the adolescent years. Beuhring and Kee (1987a, b) asked fifth and twelfth graders to talk through the strategies they used as they learned noun pairs. Fifth-graders were much more likely to repeat the pairs to themselves than to elaborate them, whereas twelth grader adolescents spontaneously preferred elaboration to simple rehearsal. The twelfth graders also relied on an arsenal of other techniques that the younger children were much less likely to use. Many of these were meaning-based, creating, for example, events that integrated the pairs, "I drank COFFEE while playing the HARP", or relying on general event knowledge, "a JANITOR wouldn't wear VELVET."

A regression analysis by Beuhring and Kee (1987b) showed that differential usage of elaboration and other associative strategies predicted the majority of the variance in the ability to recall word pairs. In other words, the greater success of the older children may be due not to an increase in memory ability itself, but to increasingly effective semantic-based learning strategies. And indeed, when younger children were told to elaborate, their recall performance more than doubled. Such instructions had very little effect on the older adolescent, given that their performance was already high because they spontaneously used such elaborative strategies.

Finally, in a study that brings together false memories and adolescent word-pair memory strategies, Odegard, Holliday, Brainerd, and Reyna (2008) investigated the finding that false recall and recognition rates are lower among children than they are for adults. To study the reasons underlying this phenomenon, Odegard et al. compared the performance of eleven-year-olds to that of young adults (with a mean age of 24) on a modified DRM task. In this paradigm, participants were shown items from a DRM list in a manner such that every item was paired with an associated word. Crucially, the associates were designed to either bias the list item's meaning toward the non-presented critical word (the context-toward condition; presenting shade-drapes in the list related to window), or away from it (the context-away condition; presenting shade-tree in the window list). Consistent with fuzzy-trace theory, Odegard et al. predicted that the children's false recognition rates would increase in the contexttoward condition because they would be more likely to encode the appropriate gist under such learning conditions.

Although there is evidence that children over the age of nine are better able to extract gist from a presented list of words compared to younger children, the former are still not as adept at this skill as are adults (Brainerd, Forrest, Karibian, \& Reyna, 2006). Thus, if false memories elicited by the DRM paradigm are due to efficient gist extraction, learning conditions that facilitate gist processing should increase the likelihood that critical items related to a list's gist trace will be falsely recognized. In fact, whereas Odegard et al. found that the type of encoding context did not alter false recognition rates for adults, false recognition of critical lures was more likely in the context-toward condition for elevenyear-olds. Thus, consistent with Rohwer et al. (1982), the eleven-year-olds significantly benefited from 
cues that facilitated the creation of meaningful connections between concepts, whereas such semantic processing took place without cuing in the older participants.

In summary, adolescents, like adults, do not learn arbitrary associations. Instead, even when tested in 'associative learning' tasks, they show a developmental increase in the ability to semantically elaborate word pairs. That is, older adolescents possess the requisite generalized knowledge of events and situations, as well as the ability to bring this knowledge to bear even in a somewhat novel situation, allowing them to find or create meaningful relations between word pairs, rather than simply attempting to learn them as unrelated word associates. These abilities increase across adolescence, as the child becomes a more sophisticated learner, on the one hand, and develops richer and more sophisticated knowledge of events and situations, on the other.

\section{Conclusions}

In this chapter, we have put forth a number of arguments concerning the relationship between association and meaning. First, association in the sense of spatial and temporal co-occurrence in the world and language is an important driving force in learning and forming semantic representations. Second, word association norms are an interesting and rich source of data. Third, word associations on their own do not provide insight into the relations that are encoded in semantic memory. Rather, word associations are driven by meaningful semantic relations. Furthermore, these relations are identifiable, and in many cases, quantifiable. Fourth, we have argued that it is not fruitful to attempt to understand semantic memory using a binary distinction between semantic similarity and word association (or even between semantic relatedness, broadly defined, vs. word association). On the one hand, the scope of semantic relations is much broader than similarity alone, and on the other, word associations are driven almost exclusively by semantic relations. Finally, a fruitful research strategy is to work toward understanding the relative importance or centrality of various types of semantic relations for various types of concepts. We highlighted a number of such investigations, and would have highlighted others were it not for space limitations. This approach, we believe, is the best path forward for understanding concepts, semantic memory, and their development.

\section{References}

Alario, F.-X., Segui, J., \& Ferrand, L. (2000). Semantic and associative priming in picture naming. Quarterly Journal of Experimental Psychology, 53A, 741-764.

Anisfeld, M., \& Knapp, M. (1968). Association, synonymity, and directionality in false recognition. Journal of Experimental Psychology, 77, 171-179.

Bajo, M.-T. (1988). Semantic facilitation with pictures and words. Journal of Experimental Psychology: Learning, Memory, and Cognition, 14, 579-589.

Berry, F. M., \& Cole, S. R. (1973). Stimulus selection in paired-associated learning: Consonant-triad versus word-triad paradigms. Journal of Experimental Psychology, 97, 402-404.

Beuhring, T., \& Kee, D. W. (1987a). Elaborative propensities during adolescence: The relationships between memory knowledge, strategy behavior, and memory performance. In M. A. McDaniel \& M. Pressley (Eds.), Imagery and Related Mnemonic Processes (pp. 257-273). New York: Springer-Verlag.

Beuhring, T., \& Kee, D. W. (1987b). Developmental relationships among metamemory, elaborative strategy use, and associative memory. Journal of Experimental Child Psychology, 44, 377-400.

Bolte, J., Jorschick, A., \& Zwitserlood, P. (2003). Reading yellow speeds up naming a picture of a banana: Facilitation and inhibition in picture-word interference. In F. Schmalhofer, R. M. Young, \& G. Katz (Eds.), Proceedings of the European Cognitive Science Conference 2003 (pp. 55-60). Mahwah, NJ: Lawrence Earlbaum Associates.

Bower, G. H. (2000). A brief history of memory research. In E. Tulving \& F. I. M. Craik (Eds.), The Oxford Handbook of Memory (pp. 3-32). New York: Oxford University Press.

Bradley, M. M., \& Lang, P. J. (1999). Affective norms for English words (ANEW): Stimuli, instruction manual and affective ratings (Tech. Rep. No. C-1). Gainesville, FL: The Center for Research in Psychophysiology, University of Florida. 
Brainerd, C. J., Forrest, T. J., Karibian, D., \& Reyna, V. F. (2006). Development of the false memory illusion. Developmental Psychology, 42, 962-979.

Brainerd, C. J., \& Reyna, V. F. (2002). Fuzz-trace theory and false memory. Current Directions in Psychological Science, 11, 164-169.

Brainerd, C. J., Yang, Y., Reyna, V. F., Howe, M. L., \& Mills, B. A. (2008). Semantic processing in "associative" false memory. Psychonomic Bulletin \& Review, 15, 1035-1053.

Burgess, C., \& Lund, K. (1997). Modelling parsing constraints with high-dimensional context space. Language and Cognitive Processes, 12, 177-210.

Cann, D. R., McRae, K., \& Katz, A. N. (in press). False recall in the Deese-Roediger-McDermott paradigm: The roles of gist and associative strength. Quarterly Journal of Experimental Psychology.

Carroll, M., \& Kirsner, K. (1982). Context and repetition effects in lexical decision and recognition memory. Journal of Verbal Learning and Verbal Behavior, 21, 55-69.

Chiarello, C., Burgess, C., Richards, L., \& Pollock, A. (1990). Semantic and associative priming in the cerebral hemispheres: Some words do, some words don't . . . sometimes, some places. Brain and Language, 38, 75 104.

Collins, A. M., \& Loftus, E. F. (1975). A spreading activation theory of semantic processing. Psychological Review, 82, 407-428.

Collins, A. M., \& Quillian, M. R. (1969). Retrieval time from semantic memory. Journal of Verbal Learning and Verbal Behavior, 8, 240-247.

Costa, A., Alario, F.-X., \& Caramazza, A. (2005). On the categorical nature of the semantic interference effect in the picture-word interference paradigm. Psychonomic Bulletin \& Review, 12, 125-131.

Crutch, S. J., \& Warrington, E. K. (2010). The differential dependence of abstract and concrete words upon associative and similarity-based information: Complementary semantic interference and facilitation effects. Cognitive Neuropsychology, 27, 46-71.

Dagenbach, D., Horst, S., \& Carr, T. H. (1990). Adding new information to semantic memory: How much learning is enough to produce automatic priming? Journal of Experimental Psychology: Learning, Memory, and Cognition, 16, 581-591.

Deese, J. (1959). On the prediction of occurrence of particular verbal intrusions in immediate recall. Journal of Experimental Psychology, 58, 17-22.

Deese, J. (1965). The structure of associations in language and thought. Baltimore, MD: Johns Hopkins Press.

Estes, Z., Golonka, S., \& Jones, L. L. (in press). Thematic thinking: The apprehension and consequences of thematic relations. Psychology of Learning and Motivation.

Ferretti, T. R., McRae, K., \& Hatherell, A. (2001). Integrating verbs, situation schemas, and thematic role concepts. Journal of Memory \& Language, 44, 516-547.

Fischler, I. (1977). Semantic facilitation without association in a lexical decision task. Memory \& Cognition, 5, 335-339.

Frenck-Mestre, C., \& Bueno, S. (1999). Semantic features and semantic categories: Differences in the rapid activation of the lexicon. Brain and Language, 68, 199-204.

Glaser, W. R., \& Dungelhoff, F.-J. (1984). The time course of picture-word interference. Journal of Experimental Psychology: Human Perception and Performance, 10, 640-654.

Goshen-Gottstein, Y., \& Moscovitch, M. (1995). Repetition priming for newly formed and preexisting associations: Perceptual and conceptual influences. Journal of Experimental Psychology: Learning, Memory, and Cognition, 21, 1229-1248.

Grossman, L., \& Eagle, M. (1970). Synonymity, antonymity, and association in false recognition responses. Journal of Experimental Psychology, 83, 244-248.

Guida, A., \& Lenci, A. (2007). Semantic properties of word associations to Italian verbs. Italian Journal of Linguistics, 19, 293-326.

Hare, M., Jones, M., Thomson, C., Kelly, S., \& McRae, K. (2009). Activating event knowledge. Cognition, 111, 151-167.

Hines, D., Czerwinski, M., Sawyer, P. K., \& Dwyer, M. (1986). Automatic semantic priming: Effect of category exemplar level and word association level. Journal of Experimental Psychology: Human Perception and Performance, 12, 370-379.

Hutchison, K. A. (2002). The effect of asymmetrical association on positive and negative semantic priming. 
Memory \& Cognition, 30, 1263-1276.

Hutchison, K. A. (2003). Is semantic priming due to association strength or feature overlap? A microanalytic review. Psychonomic Bulletin \& Review, 10, 785-813.

Jones, M. N., Kintsch, W., \& Mewhort, D. J. K. (2006). High-dimensional semantic space accounts of priming. Journal of Memory and Language, 55, 534-552.

Jung, C. G. (1919). Studies in Word-Association. (M. D. Eder, Trans.). New York: Moffatt, Yard \& Company.

Kalenine, S., Peyrin, C., Pichat, C., Segebarth, C., Bonthoux, F., \& Baciu, M. (2009). The sensory-motor specificity of taxonomic and thematic conceptual relations: A behavioral and fMRI study. NeuroImage, 44, 1152-1162.

La Heij, W., Dirkx, J., \& Kramer, P. (1990). Categorical interference and associative priming in picture naming. British Journal of Psychology, 81, 511-525.

Landauer, T. K., \& Dumais, S. T. (1997). A solution to Plato's problem: the Latent Semantic Analysis theory of acquisition, induction, and representation of knowledge. Psychological Review, 104, 211-240.

Lucas, M. (2000). Semantic priming without association: A meta-analytic review. Psychonomic Bulletin \& Review, 7, 618-630.

Lupker, S. J. (1984). Semantic priming without association: A second look. Journal of Verbal Learning and Verbal Behavior, 23, 709-733.

McKoon, G., \& Ratcliff, R. (1992). Spreading activation versus compound cue accounts of priming: Mediated priming revisited. Journal of Experimental Psychology: Learning, Memory, and Cognition, 18, 1155-1172.

McRae, K., \& Boisvert, S. (1998). Automatic semantic similarity priming. Journal of Experimental Psychology: Learning, Memory, and Cognition, 24, 558-572.

McRae, K., Cree, G. S., Seidenberg, M. S., \& McNorgan, C. (2005). Semantic feature production norms for a large set of living and nonliving things. Behavior Research Methods, 37, 547-559.

McRae, K., de Sa, V., \& Seidenberg, M. S. (1997). On the nature and scope of featural representations of word meaning. Journal of Experimental Psychology: General, 126, 99-130.

Moss, H. E., Ostrin, R. K., Tyler, L. K., Marslen-Wilson, W. D. (1995). Accessing different types of lexical semantic information: Evidence from priming. Journal of Experimental Psychology: Learning, Memory, and Cognition, 21, 863-883.

Neely, J. H., \& Durgunoglu, A. Y. (1985). Dissociative episodic and semantic priming effects in episodic recognition and lexical decision tasks. Journal of Memory and Language, 24, 466-489.

Nelson, D. L., McEvoy, C. L., \& Dennis, S. (2000). What is free association and what does it measure? Memory \& Cognition, 28, 887-899.

Nelson, D. L., McEvoy, C. L., \& Schreiber, T. A. (1998). The University of South Florida word association, rhyme, and word fragment norms. <http://web.usf.edu/FreeAssociation/>

O'Connor, C. M., Cree, G. S., \& McRae, K. (2009). Conceptual hierarchies in a flat attractor network: Dynamics of learning and computations. Cognitive Science, 33, 665-708.

Odegard, T. N., Holliday, R. E., Brainerd, C. J., \& Reyna, V. F. (2008). Attention to global gist processing eliminates age effects in false memories. Journal of Experimental Child Psychology, 99, 96-113.

Payne, D. G., Elie, C. J., Blackwell, J. M., \& Neuschatz, J. S. (1996). Memory illusions: Recalling, recognizing, and recollecting events that never occurred. Journal of Memory \& Language, 35, 261-285.

Pecher, D., \& Raaijmakers, J. G. W. (1999). Automatic priming for new associations in lexical decision and perceptual identification. Quarterly Journal of Experimental Psychology, 52A, 593-614.

Prior, A., \& Bentin, S. (2003). Incidental formation of episodic associations: The importance of sentential context. Memory \& Cognition, 31, 306-316.

Prior, A., \& Bentin, S. (2008). Word associations are formed incidentally during sentential semantic integration. Acta Psychologica, 127, 57-71.

Riordan, B., \& Jones, M. N. (2011). Redundancy in perceptual and linguistic experience: Comparing featurebased and distributional models of semantic representation. Topics in Cognitive Science, 3, 303-345.

Roediger, H. L., III, Watson, J. M., McDermott, K. B., \& Gallo, D. A. (2001). Factors that determine false recall: A multiple regression analysis. Psychonomic Bulletin \& Review, 8, 385-407.

Rohwer, W. D. Jr., Rabinowitz, M., \& Dronkers, N. F. (1982). Event knowledge, elaborative propensity, and the development of learning proficiency. Journal of Experimental Child Psychology, 33, 492-503.

Rosinski, R. R. (1977). Picture-word interference is semantically based. Child Development, 48, 643-647. 
Sailor, K., Brooks, P.J., Bruening, P.R., Seiger-Gardner, L., \& Guterman, M. (2009). Exploring the time course of semantic interference and associative priming in the picture-word interference task. Quarterly Journal of Experimental Psychology, 62, 789-801.

Santos, A., Chaigneau, S. E., Simmons, W. K., \& Barsalou, L. W. (in press). Property generation reflects word association and situated simulation. Language \& Cognitive Processes.

Schrijnemakers, J. M. C., \& Raaijmakers, J. G. W. (1997). Adding new word associations to semantic memory: Evidence for two interactive learning components. Acta Psychologica, 96, 103-132.

Seidenberg, M. S., Waters, G. S., Sanders, M., \& Langer, P. (1984). Pre- and postlexical loci of contextual effects on word recognition. Memory \& Cognition, 12, 315-328.

Shelton, J. R., \& Martin, R. C. (1992). How semantic is automatic semantic priming? Journal of Experimental Psychology: Learning, Memory, and Cognition, 18, 1191-1210.

Smith, M. C., MacLeod, C. M., Bain, J. D., \& Hoppe, R. B. (1989). Lexical decision as an indirect test of memory: Repetition priming and list-wide priming as a function of type of encoding. Journal of Experimental Psychology: Learning, Memory, and Cognition, 15, 1109-1118.

Sperber, R. D., McCauley, C., Ragain, R. D., \& Weil, C. M. (1979). Semantic priming effects on picture and word processing. Memory \& Cognition, 7, 339-345.

Thompson-Schill, S. L., Kurtz, K. J., \& Gabrieli, J. D. E. (1998). Effects of semantic and associative relatedness on automatic priming. Journal of Memory and Language, 38, 440-458.

Toglia, M. P., \& Battig, W. F. (1978). Handbook of semantic word norms. Hillsdale, NJ: Lawrence Erlbaum Assocates.

Tulving, E. (1972). Episodic and semantic memory. In E. Tulving \& W. Donaldson (Eds.), Organization of Memory (pp. 381-403). New York: Academic Press.

Vigliocco, G., Vinson, D. P., Lewis, W., \& Garrett, M. F. (2004). Representing the meanings of object and action words: The featural and unitary semantic space hypothesis. Cognitive Psychology, 48, 422-488.

Vinson, D. P., \& Vigliocco, G. (2008). Semantic feature production norms for a large set of objects and events. Behavior Research Methods, 40, 183-190.

Wu, L.-L, \& Barsalou, L. W. (2009). Perceptual simulation in conceptual combination: Evidence from property generation. Acta Psychologica, 132, 173-189.

Yee, E., Overton, E., \& Thompson-Schill, S. L. (2009). Looking for meaning: Eye movements are sensitive to overlapping features, not association. Psychonomic Bulletin \& Review, 16, 869-874. 
Table 1. Semantic Relatedness Taxonomy

\begin{tabular}{|c|c|c|}
\hline Relationship Type & Subtype & Examples \\
\hline \multirow[t]{4}{*}{ Similar Concepts } & category coordinates & fox - wolf; hammer - pliers \\
\hline & category exemplar pairs & vehicle - truck; dog - spaniel \\
\hline & synonyms & car - automobile; dawn - daybreak \\
\hline & antonyms & light - dark; good - evil \\
\hline \multirow[t]{9}{*}{ Entity } & made-of & sink - enamel; pliers - metal \\
\hline & entity behavior & clock - ticking \\
\hline & external component & tricycle - pedals \\
\hline & external surface property & apple - red \\
\hline & internal component & cherry - pit \\
\hline & internal surface property & fridge - cold, cake - sweet \\
\hline & larger whole & ant - colony \\
\hline & quantity & slippers - pair \\
\hline & systemic feature & dolphin - intelligent \\
\hline \multirow[t]{8}{*}{ Situation } & action/manner & screwdriver - turning \\
\hline & situational & saucer - teacup \\
\hline & function & drill - carpentry \\
\hline & location & cupboard - kitchen \\
\hline & origin & walnut - trees \\
\hline & patient & mop - floor \\
\hline & participant & wand - magician \\
\hline & time & turkey - Thanksgiving \\
\hline \multirow[t]{3}{*}{ Introspective } & affect emotion & wasp - annoyance; rattlesnake - fear \\
\hline & contingency & car - gasoline \\
\hline & evaluation & gown - fancy \\
\hline \multirow[t]{4}{*}{ Event } & event-agent & lecture - professor \\
\hline & event-patient & arrest - criminal \\
\hline & event-instrument & cut - knife \\
\hline & event-location & swim - lake \\
\hline
\end{tabular}


Table 2. Santos et al. (in press): A Taxonomy of Associative Relatedness

\begin{tabular}{lll}
\hline \multicolumn{2}{l}{ Type of Association } & Examples \\
\hline 1. & Compound continuation forward & baseball - bat \\
2. & Compound continuation backward & golf - miniature \\
3. & Sound similarity & nature - nurture; roar - bore \\
4. & Root similarity & convey - conveyance \\
5. & Synonyms & car - automobile \\
6. & Antonyms & light - heavy \\
7. & Domain higher level & chair - furniture \\
8. & Domain lower level & car - convertible \\
9. & Domain same level & wolf - fox \\
10. & Aspect of an object or situation & shark - teeth; restaurant - menu \\
\hline
\end{tabular}

Table 3. Studies examining semantic and associative priming, detailing criteria for semantic relatedness decision and typical examples of non-semantic associated stimuli

\begin{tabular}{|l|l|l|}
\hline Study & Semantic Relation & Example of Associated Stimuli \\
\hline Fischler (1977) & category coordinates & sugar-sweet; arm-leg; dream-sleep \\
\hline Lupker (1984) & category coordinates & beet-red; ostrich-feather; sleep-bed \\
\hline Chiarello et al. (1990) & category coordinates & cradle-baby; hammer-nail; rubber-tire \\
\hline McKoon \& Ratcliff (1992) & $\begin{array}{l}\text { based on association and } \\
\text { co-occurrence }\end{array}$ & kitchen-knife; apple-pie; officer-army \\
\hline Shelton \& Martin (1992) & category coordinates & hot-stove; day-night; cold-hot \\
\hline $\begin{array}{l}\text { Thomson-Schill et al. } \\
(1998)\end{array}$ & $\begin{array}{l}\text { Category coordinates and } \\
\text { featural data }\end{array}$ & book-worm; bus-boy; fruit-fly \\
\hline Hutchinson (2002) & $\begin{array}{l}\text { No semantic only } \\
\text { condition }\end{array}$ & piano-key; engine-car; duck-water \\
\hline
\end{tabular}

